\title{
Impactos da elevação do nível médio do mar sobre o ecossistema manguezal: a contribuição do sensoriamento remoto e modelos computacionais
}

\author{
Impacts of sea-level rise over the mangrove ecosystem: \\ the contribution of remote sensing and computer models
}

\author{
Denilson da Silva Bezerra ${ }^{1}$, Silvana Amaral², Milton Kampel ${ }^{3}$ \\ 1 Doutorando em Ciência do Sistema Terrestre pelo Instituto Nacional de Pesquisas Espaciais - INPE, Brasil \\ 2. Pesquisadora da Divisão de Processamento de Imagens do Instituto Nacional de Pesquisas Espaciais - INPE, Brasil \\ 3 - Pesquisador da Divisão de Sensoriamento Remoto do Instituto Nacional de Pesquisas Espaciais - INPE , Brasil
}

\begin{abstract}
Resumo
O processo de mudança climática resultante das atividades humanas é muito provável e dentre suas consequências, a elevação do nível médio do mar é a maior preocupação para as zonas costeiras. Devido sua posição entre o mar e o continente, o ecossistema manguezal é particularmente vulnerável às variações do mar. No Brasil, há um grande desafio no que se refere ao entendimento do padrão de resposta do manguezal a elevação do nível do mar, uma vez que a Amazônia Legal tem a maior área continua de manguezal do planeta e o país ainda apresenta a segunda maior área continua de manguezal do mundo. O presente estudo tem como objetivo discutir duas abordagens metodológicas que podem ser úteis para estudar os impactos da elevação do nível médio do mar sobre o ecossistema manguezal. As ferramentas metodológicas discutidas são: o sensoriamento remoto e modelos computacionais. Respectivamente, estas metodologias contribuem para mapeamento de florestas de mangues e simulação do padrão de resposta do manguezal a eventuais cenários de elevação do nível do mar. Ambas as abordagens metodológicas possuem vantagens e desvantagens, e podem ser utilizadas de forma conjunta para uma melhor compreensão dos impactos ao ecossistema manguezal.
\end{abstract}

Palavras-chave: Mudança climática, elevação do nível do mar, manguezal, sensoriamento remoto, modelos computacionais.

\begin{abstract}
The process of climate change resultant of human activity is very likely and among its consequences, the sea-level rise is the biggest concern for coastal zones. Due to its position between the sea and the continent, the mangrove ecosystem is particularly vulnerable to variations of the sea. In Brazil there is a big challenge with regard the understanding of the pattern of mangrove response to sea-level rise, since the Legal Amazon has the largest continuous area of mangrove of the planet, and the country still has the second largest area of mangrove forest of the world. The present study aims to discuss two methodological approaches that may be useful to study the impacts of sea-level rise over mangrove ecosystem. The methodological tools discussed are: remote sensing and computational models. Respectively, these methodologies contribute to mapping of mangrove forests and simulation of the mangrove's response pattern to eventual scenarios of sea-level rise. Both of these the methodological approaches have advantages and disadvantages, and can be used jointly for a better understanding of the impacts to the mangrove ecosystem.
\end{abstract}

Keywords: Climate change, sea-level rise, mangrove, remote sensing. 


\section{Introdução}

O processo de aquecimento do sistema climático decorrente das atividades humanas é muito provável (IPCC, 2007; 2013). Um efeito característico das mudanças climáticas na zona costeira é o fenômeno de elevação do nível médio relativo do mar (NMRM).

Dentre os impactos das mudanças climáticas, o manguezal é particularmente vulnerável ao processo de elevação do nível do mar (FIELD, 1995; LOVELOCKe ELLISON, 2007; FARACO, ANGRETO-FILHO e LANA, 2010; GIRI et al., 2011). Contudo, o padrão de resposta do manguezal à elevação do nível do mar é dependente das características geográficas, oceanográficas e ambientais, nas escalas local e regional (GILMAN et al., 2006), assim como, também, da capacidade adaptativa das espécies vegetais do manguezal (ESPINOSA e FLORES, 2011). Fato que indica a complexidade e a necessidade de haver pesquisa abordando à temática.

Pela expressiva área de ocorrência de manguezal no Brasil, entender os impactos das mudanças climáticas para articulação de medidas de preservação e mitigação é um grande desafio. Segundo SCHAEFFER-NOVELLI et al. (2000), a área de distribuição de manguezal em território brasileiro vai da fronteira com a Guiana Francesa, logo acima da linha do Equador $\left(04\right.$ o $\left.30^{\prime} \mathrm{N}\right)$ até o estado de Santa Catarina (28o 30' S). A maior área continua de manguezal do planeta encontra-se na Amazônia Legal (SOUZA-FILHO, 2005), com aproximadamente $8.900 \mathrm{~km} 2$ (KJERFV et al., 2002). Em termos de área total de manguezal, o Brasil é segundo país no mundo (SPALDING, KAINUMAAND e COLLINS, 2010; GIRI et al., 2011) com área estimada em 13.000 km2 (SPALDING, KAINUMAAND e COLLINS, 2010).

Tomando-se por base a área de manguezal no Brasil, entender os impactos das mudanças climáticas para articulação de medidas de preservação/conservação e mitigação é imprescindível. Poucos estudos têm sido direcionados para compreender e simular o padrão de resposta do manguezal às alterações climáticas em território brasileiro, entre estes é possível mencionar: Soares (2009) que desenvolveu um modelo conceitual que indica os potenciais impactos da elevação do mar no ecossistema manguezal; e FARACO, ANGRETO-FILHO e LANA (2010) que formularam uma metodologia para estimar o grau de vulnerabilidade do manguezal e populações de pescadores aos impactos oriundos da alteração no clima.

Além da importância em área de ocorrência e pela pouca produção cientifica brasileira para a temática em questão, estudar o manguezal no contexto das implicações climáticas se justifica pela forma secundária que este ecossistema tem sido submetido nos fóruns de mudanças no clima (NELLMAN et al., 2009).

Com base no conhecimento científico existente, através do presente estudo objetiva-se apresentar potenciais abordagens metodológicas de análises e monitoramento para entendimento do padrão de resposta do ecossistema manguezal à elevação do nível do mar.

Inicialmente, faz-se uma análise dos possíveis impactos da elevação do nível do mar sobre o manguezal. E posteriormente, apresentam-se abordagens metodologias que podem ser utilizadas para avaliação das respostas do manguezal em relação ao processo de elevação do nível do mar, enfocando especificamente as ferramentas disponíveis em sensoriamento remoto e no uso da modelagem computacional.

\section{Processo de Elevação do Nível do Mar e a Dinâmica do Ecossistema Manguezal}

Os sistemas terrestres têm evidenciado sinais das alterações no clima (IPCC, 2007), podendo-se mencionar, entre outras: (i) a média global do nível do mar subiu em uma taxa de 1,8 mm entre 1961 e 2003; (ii) observações realizadas desde 1961 mostram que a temperatura média do oceano aumentou em profundidades de até $3.000 \mathrm{~m}$; (iii) o oceano tem absorvido mais de $80 \%$ do calor acrescentado ao sistema climático. Pesquisas mais recentes, como a de SOLOMON et al. (2010) indicam que a gravidade das mudanças climáticas induzidas pelas emissões de dióxido de carbono $(\mathrm{CO} 2) 1$ oriundas das atividades antrópicas é irreversível por pelo menos 1.000 anos, mesmo se cessadas as atuais taxas de emissões. Isso porque a diminuição de $\mathrm{CO} 2$ na atmosférica diminui o forçamento radiativo2, contudo, tal processo é compensado pelo lento processo de expansão térmica dos oceanos.

A elevação do NMRM durante o século XX variou de $0,12 \mathrm{~m}$ a $0,22 \mathrm{~m}$ (IPCC, 2007). Outras projeções indicam que a elevação do NMRM pode oscilar de 0,26 $\mathrm{m}$ a 0,59 $\mathrm{m}$ até a última década do século XXI (SOLOMON et al., 2007). O quinto relatório de avaliação do Painel Intergovernamental de Mudanças Climáticas (IPCC-AR5) levando em consideração observações realizadas entre 1986 a 2005, indica que a taxa de elevação média global do mar pode variar de 0,45 a $0,81 \mathrm{~m}$ até as duas últimas décadas do século XXI (IPCC, 2013).

1 Principal gás de efeito estufa de origem das atividades humanas, "uso de combustíveis fósseis e das formas d e uso e cobertura do solo" (IPCC, 2007; 2013).

$2 \mathrm{O}$ forçamento radiativo é uma medida da influência de um fator na alteração do equilíbrio da energia que entra e sai do sistema Terra-atmosfera. É um índice da importância do fator como possível mecanismo de mudança do clima. O forçamento positivo tende a aquecer a superfície, enquanto o forçamento negativo tende a esfriá-la (IPCC, 2007). 


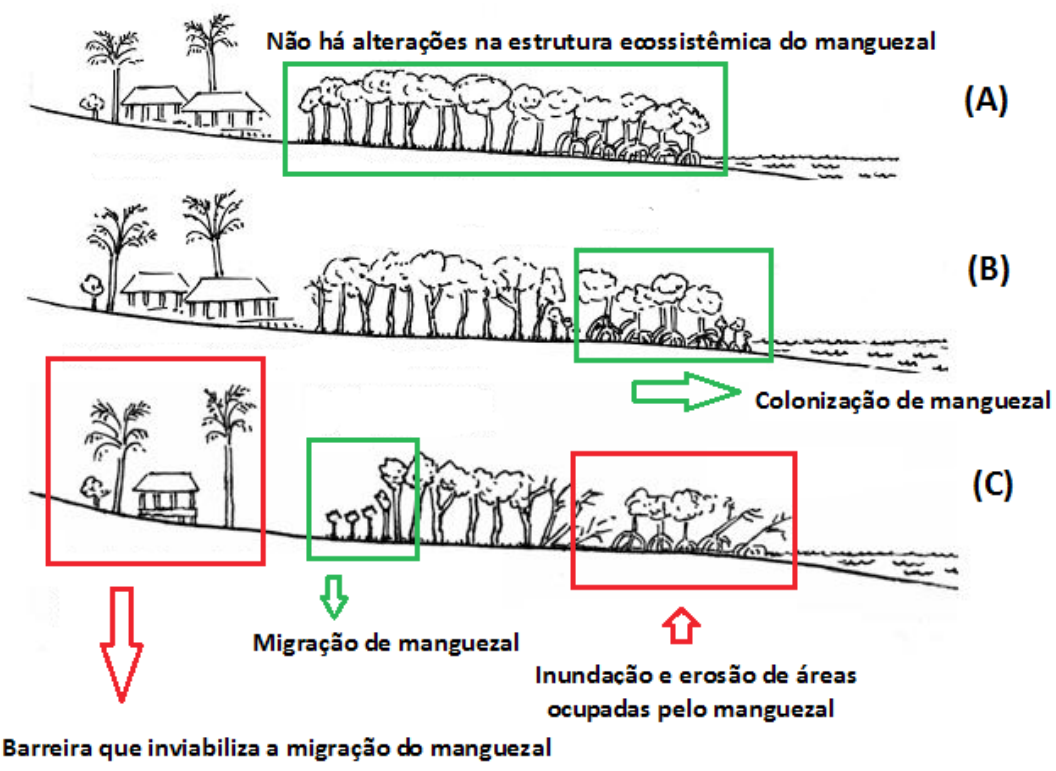

Figura 1. Cenários para o padrão geral de resposta do manguezal a alterações no NMRM: (A) - Cenário de não elevação; (B) Cenário de retração e $(C)$ - Cenário de elevação.

Fonte: adaptado de GILMAN et al. (2006)

Os impactos das mudanças climáticas que afetam o manguezal incluem elevação do nível do mar (CAHOON et al., 2006 GIRI et al., 2011), alterações na temperatura (GILMAN et al., 2008), na ocorrência de furacões (DOYLE et al., 2003), na incidência de precipitação (MCLEOD e SALM, 2006; ALONGI, 2008), nas taxas de concentrações de CO2 (MCLEOD e SALM, 2006), alterações no padrão de circulação oceânica (GILMAN et al., 2008; SOARES 2009) e até nas respostas humanas3 às alterações no clima (GILMAN et al., 2008).

O manguezal é particularmente suscetível ao processo de elevação do nível do mar (FIELD, 1995; LOVELOCK e ELLISON, 2007). Contudo, o ecossistema manguezal apresenta resiliência a este processo (GILMAN et al., 2006), sobretudo os manguezais localizados em locais submetidos a elevada variação de maré e que não possuam baixo relevo (MCLEOD e SALM, 2006) e também, a resposta do manguezal pode apresentar diferenças de um local para outro, como consequência de características locais e regionais onde o mesmo ocorra, tais como: padrões locais nas taxas da elevação do nível do mar e nos processos de sedimentação/erosão (ALONGI 2008; GILMAN et al., 2008), assim como, da altura de maré local, topografia do relevo e das formas de uso e ocupação do solo adjacentes ao manguezal (MCLEOD e SALM, 2006), fato que evidencia a complexidade inerente à temática.

Há três cenários gerais que definem o padrão de resposta do manguezal no que tange possíveis

3 Entende-se por "respostas humanas", obras de engenharia (diques, processo de canalização, etc.) construídas com intuito de minimizar os impactos das mudanças climáticas e que podem ocasionar impactos negativos ao manguezal (GILMAN et al., 2008). alterações no NMRM (GILMAN et al., 2006), a saber: (i) cenário de não elevação, (ii) retração do NMRM e (iii) elevação do NMRM; tendo-se em consideração uma escala local/região e um período de tempo da ordem de décadas ou mais (Figura1).

Cenário de não elevação - Quando não há aumento do nível do mar em relação à superfície de ocorrência do manguezal. Neste cenário, não há alterações significativas na estrutura ecossistêmica local do manguezal (alteração na área, por exemplo), a ocorrência e distribuição das espécies de angiospermas características do manguezal são condicionadas pelo processo de zonação pré-existente no local. Figura 1a;

Retração do NMRM - Quando ocorre retração do NMRM em relação à superfície de ocorrência do manguezal. Para este cenário, o manguezal pode ocupar áreas que outrora estavam submersas, e dependendo da topografia, o manguezal também pode expandir lateralmente. Figura $1 \mathrm{~b}$;

Aumento do NMRM - Quando ocorre aumento do NMRM em relação à superfície de ocupação do manguezal. Neste cenário, pode haver inundação permanente e erosão de áreas coupadas pelo manguezal, deslocamento da área de influência das marés, o que pode ocasionar perdas de diversidade biológica em decorrência de alterações no hidroperiodo e na zonação das espécies vegetais do manguezal, e pode ocorrer também, migração do manguezal em direção ao continente, caso não existam barreiras à sua colonização (ex. construções antrópicas). Figura 1c.

Para o cenário de elevação do NMRM, que é o foco da presente pesquisa, o manguezal pode 
sofrer diferentes alterações ambientais, tais como modificação na área de ocorrência (aumento ou diminuição), migração para áreas adjacentes, ou ainda tornar-se suscetível à perda de diversidade biológica, o que pode comprometer os bens e serviços usualmente oferecidos pelo manguezal à zona costeira (MCLEOD e SALM, 2006; FIELD, 1995; LOVELOCK e ELLISON, 2007).

A perda de diversidade biológica pode ocorrer porque a distribuição das espécies do manguezal (zonação) responde ao gradiente das marés em relação ao relevo local (LUGO, 1980), do ponto de menor valor (baixamar) até o patamar das maiores marés (preamar), a denominada área de influência das marés (AIM). Com a elevação do mar pode haver o deslocamento da AIM, e com isso o padrão inicial da zonação das espécies pode ser alterado, com possíveis mudanças na diversidade biológica, pois algumas espécies podem adaptar-se melhor que outras (AGRAWALA et al., 2003).

Ao modificar o padrão das marés, alterações no nível do mar podem também modificar o processo de sedimentação principalmente de sedimentos finos que são transportados em suspensão na coluna d'água através da ação das marés. Podendo favorecer assim a acreção de sedimento, fato que propicia aumento do substrato lamoso, o que pode favorecer a expansão do manguezal (BATISTA et al., 2008). Além da influência nos padrões de circulação de massas d'água e do transporte de sedimentos, as taxas de sedimentação podem depender também do sistema radicular de cada espécie de manguezal presente (CAHOON et al., 2006).

Por responder à dinâmica do NMRM, o ecossistema de manguezal tem sido sugerido como indicador do processo de elevação do nível do mar (SCHAEFFER-NOVELLI, CONTRÓN-MOLERO e SOARES, 2002). Outra possível resposta é a migração do manguezal para áreas adjacentes, quando a elevação do nível do mar propicia o aumento da entrada de água salgada em rios e estuários (SOARES 2009). Contudo, o processo de migração depende das formas de uso e ocupação do solo em áreas marginais ao manguezal, principalmente as de origem antrópica, que podem atuar como barreiras à colonização de novas áreas (MCLEOD e SALM, 2006; FARACO, ANGRETO-FILHO; LANA, 2010).

A elevação do nível do mar pode também propiciar redução de áreas de manguezal como observado por LARA e COHEN (2003). No município de Bragança, estado do Pará, os autores analisaram a taxa de retração de área do manguezal na área de estudo por influência da invasão de areia transportada pela coluna d'água que ao cobrir as camadas de lama causavam asfixia das raízes do manguezal. De acordo com os autores, a perda da cobertura vegetal do manguezal correspondeu a um padrão associado a uma tendência de longa duração, compatível com as taxas de elevação do nível do mar descritas pela literatura. O manguezal também pode perder área quando de um lado o manguezal é submerso e/ ou sofre com a erosão proveniente da elevação do NMRM e do outro, o mesmo não encontra condições adequadas à colonização em áreas circunvizinhas (Figura 1c).

A elevação do nível do mar aliada a alterações nas taxas de precipitação podem também modificar a salinidade do estuário, com consequências para a manutenção do manguezal. $\mathrm{O}$ aumento excessivo de salinidade pode ocasionar a morte de indivíduos vegetais ou sua adaptação às novas condições (AGRAWALA et al., 2003).

Além dos processos relacionados à elevação do nível do mar como inundação, transporte de sedimento e mudanças na salinidade, alterações nos processos de circulação oceânica também podem causar impactos ao manguezal. Porém, estes são pouco descritos na literatura, o que reflete uma lacuna significativa de conhecimento em relação ao entendimento do padrão de resposta do manguezal às alterações climáticas (ALONGI, 2008).

Embora o manguezal seja aparentemente um ecossistema simples em termos de diversidade de espécies vegetais, sua ecologia é extremamente complexa (LUGO e SNEDAKER, 1974). Inúmeras interações existem entre o manguezal e os sistemas oceânico, continental, atmosférico e até mesmo antrópico podem ser alteradas pelas mudanças climáticas, sobretudo pela elevação do NMRM: (i) a dinâmica da geofísica das marés que tem variações de horas, dias e até mesmo de meses, com reflexo direto na zonação de espécies de mangues; (ii) o aporte de água doce que ocorre mediante a ação da precipitação e da descarga de rios e água subterrânea influencia na entrada de sedimentos e nutrientes que chegam ao ecossistema; (iii) a influência das correntes marinhas determinam a deposição de sedimentos, nutrientes e o aporte de água salgada; (iv) formas de usos antrópicos diretos e indiretos podem proporcionar maior ou menor grau de vulnerabilidade ao manguezal, uma vez que as construções humanas na zona costeiras podem causar impactos ao manguezal, e também, podem se constituir como barreias a colonização de novas áreas de manguezal. 


\section{Métodos de Análise e Monitora- mento Para Áreas de Manguezais Impactadas Pela Elevação do Nível Do Mar}

Devido à natureza complexa das relações ecológicas do manguezal com seu meio físico-climático mencionado no parágrafo acima, pode-se inferir que o diagnóstico de impactos da elevação do nível do mar não é procedimento trivial, fato que evidencia a necessidade de haver uma abordagem multidisciplinar. Neste contexto, apresentam-se a seguir duas abordagens metodológicas possíveis para análise do manguezal na busca de uma melhor compreensão das respostas do mencionado ecossistema: uso de dados e técnicas de sensoriamento remoto e o uso de modelos computacionais.

\subsection{Contribuição do Sensoriamento Remoto}

Os últimos relatórios de avaliação das mudanças no clima do Painel Intergovernamental de Mudanças Climáticas - IPCC têm demonstrado a importância do uso de imagens de satélites para mensuração da elevação do NMRM em todo o planeta, assim como os potenciais impactos para a zona costeira mundial (IPCC, 2007; 2013). No que tange especificadamente os impactos da elevação do nível do mar sobre o ecossistema manguezal, o sensoriamento remoto pode, por exemplo, ser utilizado na mensuração de alterações na sua área de ocorrência como resposta às alterações no avanço do mar sobre o continente (LARA e COHEN, 2003, por exemplo).

A ferramenta de sensoriamento remoto em estudos direcionados ao ecossistema manguezal pode ser utilizada em distintas escalas (espacial, temporal e espectral) e para várias utilidades, tais como: mapeamento de áreas de ocorrência, mensuração da área foliar, identificação do padrão de resposta espectral das distintas espécies, confecção de mapas de espécies que compõem os bosques de mangues e estimação de biomassa (HEUMANN, 2011; KUENSER et al., 2011).

Os dados ópticos mais utilizados em estudos de bosques de mangues são os provenientes de aerofotogrametria e dados orbitais de media e alta resolução como as imagens Landsat e SPOT (NEWTON et al., 2009). Segundo HEUMANN (2011), algumas técnicas de sensoriamento remoto mais frequente em pesquisas cujo objeto analisado é o manguezal são: interpretação visual, classificação digital, classificação não supervisionada, classificação supervisionada, classificação híbrida, classificação espectral e índices espectrais de vegetação. A Figura 2 apresenta um exemplo de mapeamento de manguezal por sensoriamento remoto com auxílio da técnica de classificação híbrida.

Além de mapeamento, o uso de técnicas de sensoriamento remoto tem permitido mensurar outros parâmetros importantes para a compreensão das intera-

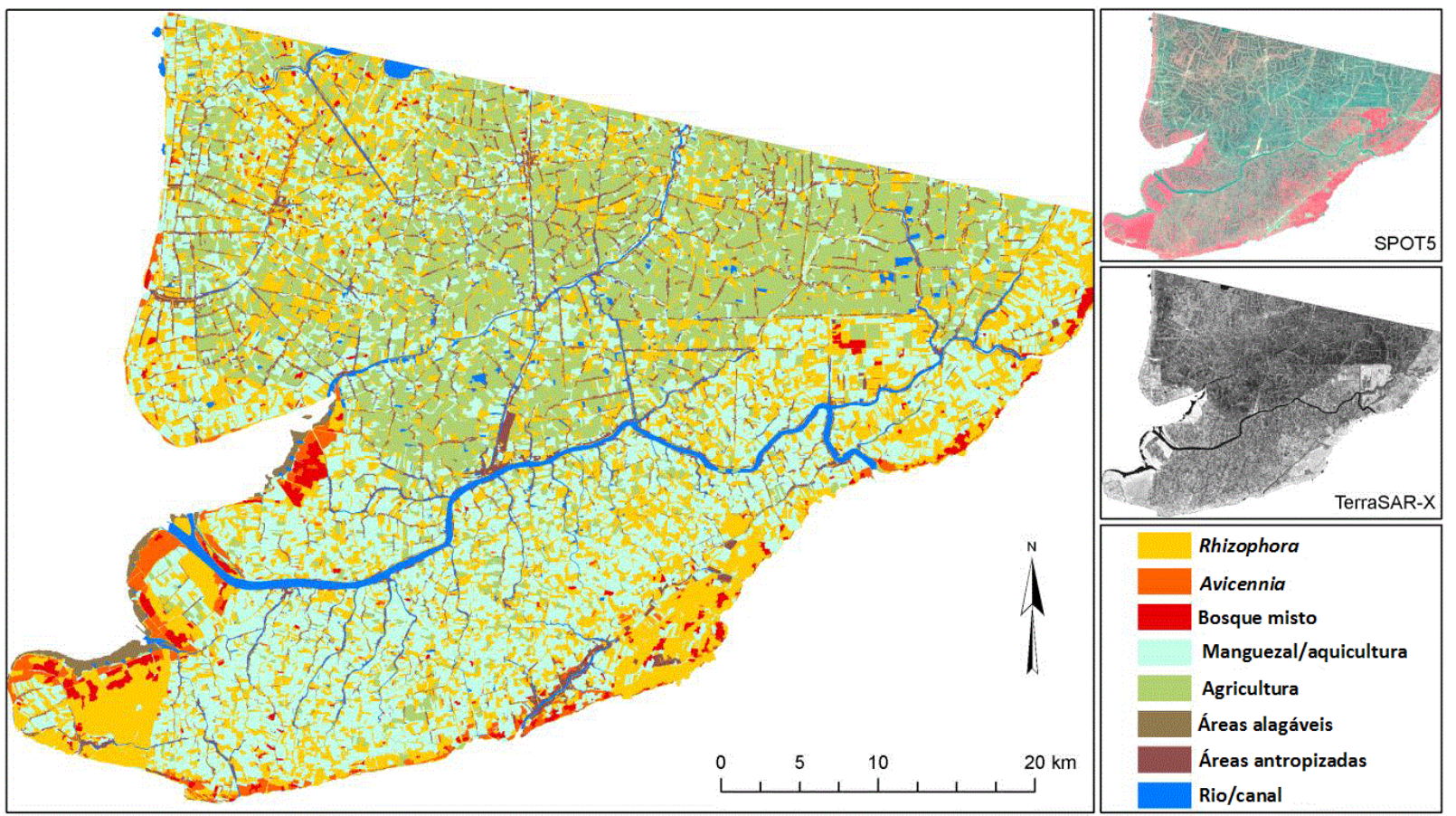

Figura 2 - Exemplo de mapeamento de áreas de manguezal utilizando o método de classificação hibrida com imagens SPOT 5 e dados de TerraSAR-X para a provincia Ca Mau no Vietinã (2010). Baseados em dois conjuntos de dados: composição de espécies e diferentes formas de uso e ocupação em áreas de ocorrência de manguezal.

Fonte: KUENSER et al. (2011) 
ções do manguezal com os sistemas físico-climáticos adjacentes, como nos casos das estimativas de carbono, áreas foliar, biomassa, identificação de espécies e altura do dossel (RASOLOFOHARINORO et al., 1998; KOVACS, WANG e FLORES-VERDUGO, 2005; FATOYINBO et al., 2008; HUANG, ZHANG e WANG, 2009) .

Muitos podem ser os usos da ferramenta de sensoriamento remoto, conduto a referida abordagem metodológica pode apresentar algumas desvantagem/ limitações, tais como: presença de nuvens, no caso de imagens ópticas, que podem prejudicar a qualidade das imagens; eventual falta de recursos humanos qualificados; em alguns casos, possíveis custos elevados para aquisição de equipamentos e softwares específicos. Outro potencial fator limitante reside no fato que o sensoriamento remoto é adequado para identificação dos "efeitos externos" dos impactos da elevação do mar (diminuição da área de manguezal, por exemplo), mas não consegue fornecer informações detalhadas sobre eventuais alterações ecológicas (ex.: processo de sucessão ecológica).
As vantagens do uso do sensoriamento remoto encontram-se na versatilidade de dados disponíveis em diferentes escalas para mapear o manguezal; na comodidade e na economia de tempo, pois tendo acesso às imagens, o pesquisador pode mensurar alterações no manguezal em diferentes anos do seu próprio laboratório; na grande disponibilidade de imagens de baixa, média e de alta resolução para todo o mundo e pela existência dados de diferentes naturezas, como imagens de radar que ampliam a capacidade de detectar características específicas dos bosques de mangue.

Outra vantagem que pode ser conferida à utilização do sensoriamento remoto reside no fato que com o seu uso é possível obter/mensurar dados reais de uma determinada área em estudo e com isso, é possível obter um registro histórico da área de ocorrência do manguezal. Fato que pode subsidiar pesquisas mais especificas que possam promover uma melhor compreensão do (s) fator (es) que pode (m) ocasionar eventuais alterações na área de manguezal presente em um determinado local.

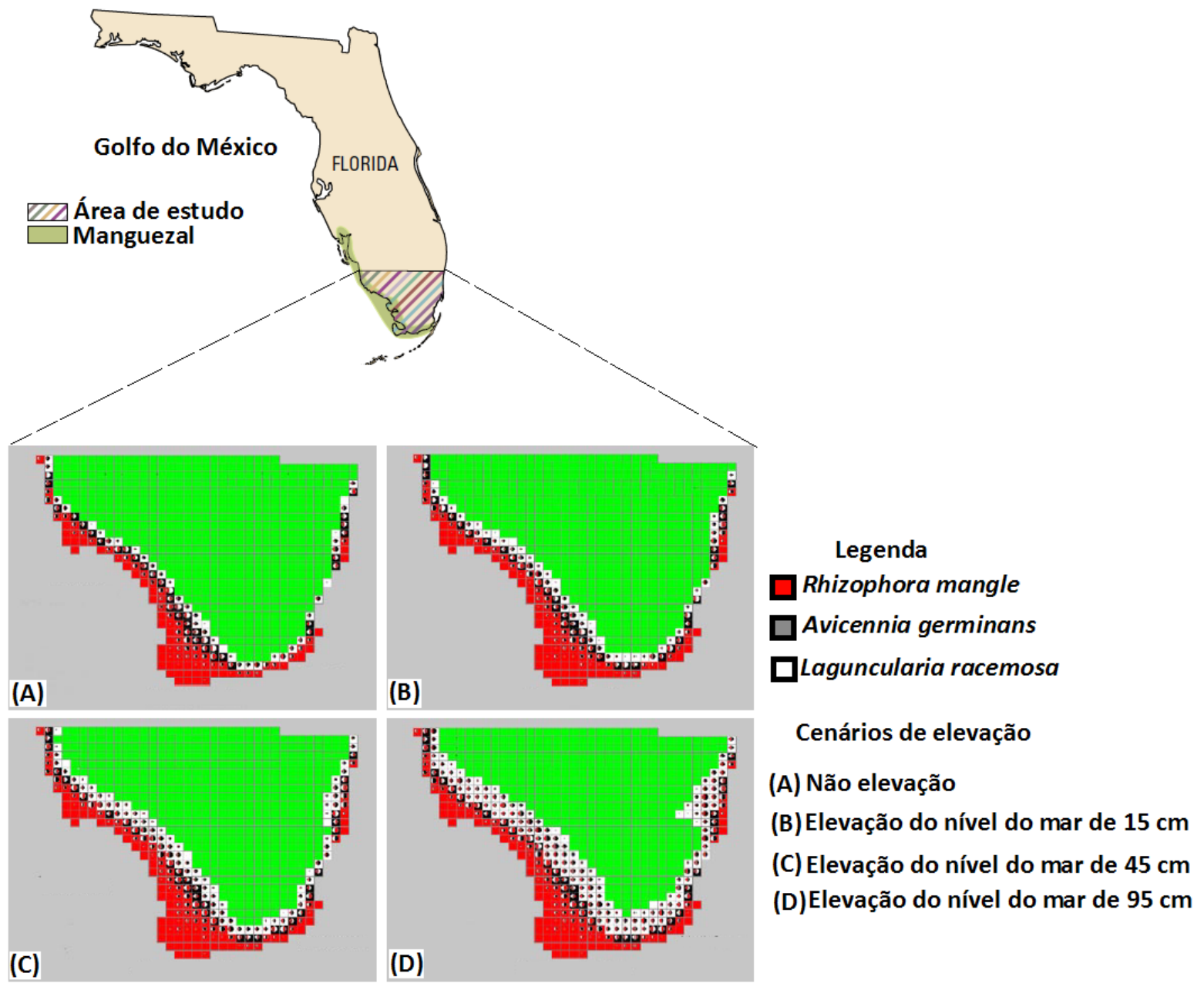

Figura 3. Modelo SELVA MANGRO sendo utilizado para simulação de processos de sucessão ecológica para três espécies de mangues como resposta a diferentes cenários de elevação do nível do mar no sul da Flórida/EUA.

Fonte: Adaptado de DOYLE, GIROD e BOOKS (2003). 


\subsection{Contribuição da Modelagem Computacional}

O uso de modelagem computacional constitui-se como uma ferramenta útil para entendimento dos impactos da elevação do nível do mar no manguezal, considerando-se duas aplicações principais: a simulação do padrão de resposta do manguezal frente aos impactos da elevação do nível do mar, antes mesmos que estes se manifestem em uma determinada área e a simulação de potenciais alterações nos processos ecológicos.

Berger et al. (2008) sustentam que a abordagem da modelagem é adequada para avaliar simultaneamente os efeitos das mudanças ambientais e alterações nos processos ecológicos tais como recrutamento, estabelecimento, crescimento, produtividade e mortalidade de indivíduos arbóreos. Em seu estudo, são discutidos três modelos - FORMAN, KIWI e MANGRO, parametrizados4 para avaliar a dinâmica do manguezal conforme descrito pela literatura para espécies de mangues neotropicais (BERGER; HILDENBRANDT, 2000; DOYLE, GIROD; BOOKS, 2003; BERGER et al., 2006).

Os modelos FORMAN, KIWI e SELVA MANGRO são modelos baseados em indivíduos (MBI). Os MBI's por serem considerados como ferramentas adequadas para simular a variabilidade de plantas ou de animais e suas influências em sistemas naturais, tornaram-se amplamente aceitos em estudos de ecologia numérica na década de 90 (GRIMM, 1999; DEANGELIS; MOOIJ, 2005). Nestes modelos, há integração hierárquica em diversos níveis de processos ecológicos que podem ser parametrizados diretamente e de forma relativamente simples. Além disso, os MBI's podem ser projetados para funcionar em diferentes escalas temporais e espaciais.

Tanto o FORMAN, KIWI, como o SELVA MANGRO foram desenvolvidos para o entendimento de florestas de mangues em diferentes condições ambientais e de manejo. Os três modelos foram parametrizados para características biológicas e fisiológicas de três espécies de mangues neotropicais: Rhizophora mangle (mangue vermelho), Avicennia germinans (mangue preto) e Laguncularia racemosa (mangue branco). Os modelos FORMAN e KIWI são mais robustos para a simulação da dinâmica dos bosques de mangues, utilizados para descrever processos ecológicos e de estrutura do manguezal (BERGER e HILDENBRANDT, 2000; BERGER et al., 2006; BERGER et al., 2008). Enquanto o SELVA MANGRO é mais direcionado à simulação da dinâmica de paisagem e

\footnotetext{
${ }^{4}$ Parametrização é um processo de obtenção de aproximações matemáticas que possam minimamente descrever o comportamento de determinadas variáveis ecossistêmicas (SANDS e LANDSBERG, 2002).
}

para grandes escalas espaciais, sendo pra isso conectado a um banco de dados geográfico.

Dentre os três modelos mencionados nos parágrafos acima, apenas o MANGRO foi utilizado para simular o padrão de resposta do manguezal às mudanças climáticas, mais precisamente para avaliar os impactos de furacões (DOYLE; GIROD, 1996) e do processo de elevação do nível do mar (DOYLE, GIROD e BOOKS, 2003), Figura 3.

A Figura 3 evidencia um exemplo de utilização do SELVA MANGRO para a simulação de possíveis alterações na composição de três espécies de manguezais no sul da Flórida/EUA como resposta a um cenário de elevação do nível do mar que oscila de 0,15 a 1,1 m, tendo-se por base um período de tempo de 1940 até meados de 2100. Em estudos como o apresentado na Figura 3, é possível se antecipar aos possíveis impactos negativos ao manguezal (perda de diversidade biológica, por exemplo), e com isso, medidas mitigadoras podem ser idealizadas pelos tomadores de decisão de forma antecipada, fato que evidencia a relevância do uso da modelagem computacional para a temática abordada.

A modelagem computacional, ao simular os impactos da elevação do nível do mar, tende a possibilitar o diagnóstico antecipado do padrão de resposta do manguezal. Como em um laboratório virtual, pode-se, por exemplo, simular alterações na salinidade, no deslocamento da AIA, nas taxas de transporte de sedimento, nos processos de sedimentação e erosão e em possíveis alterações no aporte de sedimento, e com isso, é possível se inferir sobre o padrão de resposta do manguezal à elevação do nível do mar, tendo-se por base além da questão climática, as características geográficas e ambientais da região que o manguezal coloniza, assim como, também, de áreas adjacentes que podem atuar como barreias ou como áreas aptas a serem colonizadas quando houver variação do NMRM (ver Figura 1).

Em termos de limitações é possível mencionar que por mais que seja sofisticado um modelo computacional, este se trata apenas de uma simplificação do sistema ecológico em estudo e em muitos casos a falta de dados de campo ou ainda a distribuição rara e/ou pontual dos mesmos, dificulta um processo de parametrização eficiente, o que pode reduzir a capacidade preditiva (generalidade) e a aderência à realidade, ou seja, o resultado obtido sempre será apenas uma simulação e não um dado medido. Além disso, a complexidade da dinâmica dos processos ecológicos do manguezal com o meio físico onde o mesmo ocorre pode dificultar a formulação de artifícios matemáticos de simplificação para os procedimentos de modelagem, podendo comprometer assim o caráter de precisão dos modelos. 


\section{Considerações Finais}

O manguezal é um ecossistema costeiro vulnerável ao fenômeno de elevação do nível do mar, devido sua localização entre o mar e o continente. Neste contexto, há no Brasil um grande desafio haja vista sua extensa área de manguezal, principalmente na maior área continua de manguezal do planeta, situada no perímetro da Amazônia Legal brasileira, assim como, também, devido a pouca produção cientifica brasileira voltada para a antecipação e prevenção dos potenciais impactos do aumento do nível do mar.

Foram apresentadas duas abordagens metodológicas para estudo do manguezal frente à ameaça da elevação do nível médio do mar: o sensoriamento remoto e a modelagem computacional. Todas duas apresentam potenciais distintos e complementares para fornecer informações e/ou dados que podem subsidiar atividades que visem avaliação e recuperação dos impactos decorrentes do processo de elevação do nível do mar. Apesar de fundamentadas em premissas distintas, estas abordagens podem ser utilizadas de modo complementar para o acompanhamento e até antecipação à ocorrência dos possíveis impactos.

Ambas as metodologias apresentam vantagens e desvantagens/limitações no caso do sensoriamento remoro as vantagens residem na grande disponibilidade de dados, na versatilidade de escalas que podem ser adotadas (temporal, espectral e espacial), na possibilidade de se obter dados reais (medidos), etc. Limitações e/ou desvantagem podem ser observadas no que se refere a eventuais custos elevados para aquisição de imagens e softwares especifico, possível falta de recursos humanos qualificados, dentre outros.

O uso da modelagem também apresenta desvantagens e potencialidades, entre os aspectos positivos pode-se mencionar: a possibilidade de se antecipar a ocorrência do impacto, simular alterações na área de manguezal e em seus processos ecológicos, simular o padrão de resposta dos distintos bosques de mangues, etc. Em termos de desvantagens, é possível mencionar que o resultado obtido sempre será uma simulação e não um dado medido, a eventual falta de dados locais que podem fragilizar a parametrização do modelo, a dificuldade de se obter simplificação matemática dos processos ecológicos do ecossistema manguezal, etc.

Com intuito de minimizar as desvantagens e maximizar as potencialidades, ambas as abordagens metodológicas podem ser utilizadas de forma cojunta. Como exemplo de uma abordagem complementar entre as duas ferramentas discutidas, pode-se mencionar um cenário onde um manguezal de uma determinada área foi impactado por sucessivos cenários de elevação do nível do mar ao longo de certo período de tempo. Neste caso, o sensoriamento remoto pode ser utilizado para mensurar a variação de área do manguezal que historicamente existia na área analisada. Com base nos dados disponibilizados pelo sensoriamento remoto, pode-se inferir o estado de ocupação dita "normal" do manguezal, e com este cenário de ocupação inicial, potenciais alterações no manguezal podem ser simuladas através do uso da modelagem computacional, como exemplo de estudo já realizado neste contexto pode-se mencionar a pesquisa de DI NITTO et al. (2013), onde os autores utilizaram mapeamentos pré-existentes do manguezal que serviram de base para os procedimentos de simulação computacional na área de estudo para distintos cenários de elevação do nível do mar.

\section{Agradecimento}

Os autores do presente estudo agradecem a Coordenação de Aperfeiçoamento de Pessoal de Nível Superior (CAPES) pela concessão da bolsa de doutorado que subsidiou apoio financeiro em todas as etapas da pesquisa, cujo um dos resultados é o presente artigo.

\section{Referências}

AGRAWALA, S. et al. Development and Climate Change In Fiji: Focus on Coastal Mangroves. COM/ENV/EPOC/DCD/DAC, 56 p, 2003.

ALONGI, D. M. Resilience of Mangrove Forests, Tsunamis, and Global Climate Change. Estuarine, Coastal and Shelf Science, volume 76, issue: 1, Publisher Elsevier, p. 1-13, 2008. doi:10.1016/j. ecss.2008.08.024.

\section{BATISTA, E. M.; SOUZA-FILHOP.W.M.;} SILVEIRA, O. F. M. Avaliação de Áreas Deposicionais e Erosivas em Cabos Lamosos da Zona Costeira Amazônica Através da Análise Multitemporal de Imagens de Sensores Remotos. Revista Brasileira de Geofísica, 27 (supl.1): p. 83-86, 2009.

BERGER, U.; HILDENBRANDT, H. A New Approach to Spatially Explicit Modelling of Forest Dynamics: Spacing, Ageing and Neighborhood Competition of Mangrove trees. Ecological Modelling. 132: p. 287-302, 2000.

BERGER, U. et al. Modelling Secondary Succession of Neotropical Mangroves: Causes and Consequences of Growth Reduction in Pioneer 
Species. Perspectives in Plant Ecology, Evolution and Systematic. 7: p. 243-252. doi:10.1016/j. ppees.2005.08.001, 2006

BERGER, U. et al. Advances and limitations of individual-based models to analyze and predict dynamics of mangrove forests, Aquat. Bot, 2008. doi:10.1016/j.aquabot.2007.12.015.

CAHOON, D. R. et al. Coastal wetland vulnerability to relative sea-level rise: wetland elevation trends and process controls. In: VHOEVEBN J.T.A. et al. (Eds.), Wetlands and Natural Resource Management. Ecological Studies, vol. 190. Springer-Verlag, Berlin/Heidelberg, pp. 271-292, 2006.

CORNEJO, R. H. Remote Sensing and Ethnobotanical Assessment of The Mangrove Forest Changes in The Navachiste-San IgnacioMacapule Lagoon Complex, Sinaloa, Mexico. Ecology and Society 10: 16 [online]. 2005.

DEANGELIS, D. L.; MOOIJ, W. M. Individualbased modeling of ecological and evolutionary processes. Annu. Rev. Ecol. Evol. Syst. 36, p. 147-168, 2005.

DI NITTO, D. et al. Mangroves facing climate change: landward migration potential in response to projected scenarios of sea level rise, Biogeosciences Discuss., 10, p. 3523-3558, doi:10.5194/bgd-10-3523-2013, 2013.

DOYLE, T. W.; GIROD, G. F. The frequency and intensity of Atlantic hurricanes and their influence on the structure of south Florida mangrove communities. In: H. DIAZ \& R. PULWARTY, eds., Hurricanes, Climatic Change and Socioeconomic Impacts: A Current Perspective, p. 111 128: New York, Westview Press, p. 325, 1996.

DOYLE, T.W.; GIROD, G.F.; BOOKS, M.A. Chapter 12: modeling mangrove forest migration along the southwest coast of Florida under climate change. In: NING, Z.H., TURNER, R.E., DOYLE, T.W., ABDOLLAHI, K. (Eds.), Integrated Assessment of the Climate Change Impacts on the Gulf Coast Region. GRCCC and LSU Graphic Services, Baton Rouge, LA, p. 211-221, 2003.
ERFTEMEIJER, P. L. A. A new technique for rapid assessment of mangrove degradation: A case study of shrimp farm encroachment in Thailand. Trees Structure and Function 16: p. 204-208, 2002.

ESPINOSA, L.Y.; FLORES, J. A Review of SeaLevel Rise Effect on Mangrove Forest Species: Anatomical and Morphological Modifications. In: Global Warming Impacts - Case Studies on the Economy, Human Health, and on Urban and Natural Environments. CASALEGNO, S. (ed). Published by InTech Janeza Trdine 9, 51000 Rijeka, Croatia. p. 253-276, 2011.

FATOYINBO, T.E. et al. Landscape-scale extent, height, biomass, and carbon estimation of Mozambique's mangroves forests with Landsat EMT+ and Shuttle Radar Topography Mission elevation data. Journal of Geophysical Research, Vol. 113, 2008. G02S06, doi:10.1029/2007JG000551.

FARACO, L. F. D.; ANGRETO-FILHO, J. M.; LANA, P. C. Methodology for Assessing the vulnerability of mangroves and fisherfolk to climate change. Pan-American Journal of Aquatic Sciences, 5(2): p. 205-223, 2010.

FIELD, C. Impacts of expected climate change on mangroves. Hydrobiologia 295, p. 75-81, 1995.

GILMAN, E.L. et al. Adapting to Pacific Island mangrove responses to sea level rise and climate change, Climate Research, 32, (3) p. 161-176, 2006. ISSN 0936-577X.

GILMAN, E.L. et al. Threats to mangroves from climate change and adaptation options, Aquat. Bot., 89, p. 237-250, 2008. doi:10.1016/j. aquabot.2007.12.009.

GIRI, C. et al. Status and Distribution of Mangrove Florests of the World Using Observation Satellite Data. Global Ecology and Biogeography 20, p. 154-159, 2011. doi: 10.1111/j.1466-8238.2010.00584.x.

GRIMM, V. Ten years of individual-based modelling in ecology: what have we learned and what couldwe learn in the future? Ecol. Model. 115, p. 129-148, 1999. 
HEUMANN, B.W. Satellite Remote Sensing of Mangrove Forests: Recent Advances and future opportunities. Progress in Physical Geography 35 (1): p. 87-108, 2011.

HUANG, X.; ZHANG, L.; LE WANG. Evolution of Morphological Texture Features of Mangrove Forest Mapping and Species Discrimination Using Multispectral IKONOS Imagery. IEEE Geoscience and Remote Sensing Letters, Vol. 6, No. 3, July 2009.

IPCC: Contribution of Working Group II to the Fourth Assessment Report of the Intergovernmental Panel on Climate Change. M.L. PARRY, O. F. CANZIANI, J. P. PALUTIKOF, P. J. VAN der LINDEN.; C. E. HANSON, Eds. Cambridge University Press, Cambridge, UK. 976 p, 2007.

IPCC: Working group I contribution to the IPCC fifth assessment Report (AR5), climate change 2013: the physical science basis. Coordinating Lead Authors: THOMAS STOCKER (Switzerland), QIN DAHE (China), GIAN-KASPER PLATTNER (Switzerland). 2216 p, 2013.

KOVACS, J.; WANG, J.; FLORES-VERDUGO, F. MAPPING Mangrove Leaf Area Index at the Especies Level Using IKONOS and LAI-2000 Sensors for the Agua Brava Lagoon, Mexican Pacific. Estuarine, Coastal and Shelf Science 62: p. 377-384, 2005.

KJERFV, B. Morphodynamics of muddy environments along the Atlantic coasts of North and South America. In: Muddy Coasts Of The World: Processes, Deposits and Functions. 1s ed., Amsterdam, Elsevier Science. p. 479-532, 2002.

KUENZER, C. Remote Sensing of Mangrove Ecosystems: A Review. Remote Sens 3, p. 878-978, 2011.

LAFFOLEY, D.d'A; GRIMSDITCH, G. (eds). Mangroves. The Management of Natural Coastal Carbon Sinks. D.d.A. Laffoley and G. Grimsditch. Gland Switzerland, IUCN: p. 53 pp, 2009.

LARA, R. J.; COHEN, M. C. L. Sensoriamento remoto. In: Os manguezais da costa Norte Brasileira. MARCUS, E. B. F. (org) 1a ed. Maranhão: Fundação Rio Bacanga, p.13-30. 2003.
LOVELOCK, C. E.; ELLISON, J. C. Vulnerability of mangroves and tidal wetlands of the great barrier reef to climate change. In: JOHNSON, J.E., MARSHALL, P.A. (Eds.), Climate Change and the Great Barrier Reef: A Vulnerability Assessment. Great Barrier Reef Marine Park Authority and Australian Greenhouse Office, Australia, pp. 237-269, 2007.

LUGO, A.E.; SNEDAKER, S.C. The Ecology of Mangroves. Annual Review of Ecology and Systematics, Vol. 5, p. 39-64, 1974.

LUGO, A.E. Mangrove ecosystems: successional or steady state? Biotropica 12: 65-73, 1980.

MCLEOD, E e SALM, R.V. 2006. Managing Mangroves for Resilience to Climate Change. IUNC, Gland, Switzerland. 64 p. 2006.

MURRAY, B.C. et al. Payments for blue carbon: Potential for protecting threatened coastal habitats. Nicolas Institute Policy Brief, 2010. 8 p.

NELLMANN, C. et al. (Eds). Blue carbon. A rapid response asessment, United Nations Environment Programme. GRID-Arendal, 2009. ISBN: 978-827701-060-1, www.grida.no.

NEWTON, A.C. et al. Remote sensing and the future of landscape ecology. Progress in Physical Geography 33: p. 528-546, 2009.

RASOLOFOHARINORO, M. et al. A remote sensing based methodology for mangrove studies in Madagascar. International Journal of Remote Sensing 19: p. 1873-1886, 1998.

REBELO-MOCHEL, F.; PONZONI, F. J.

Spectral characterization of mangrove leaves in the Brazilian Amazonian coast: Turiaçu Bay, Maranhão State. Annals of the Brazilian Academy of Science.79 (4): p. 683-692, 2007.

SANDS, P.J.; LANDSBERG, J.J. Parameterisation of 3-PG for plantation grown Eucalyptus globulus. Forest Ecology and Management, 163:273-292, 2002.

SCHAEFFER-NOVELLI, Y. et al. Brazil mangroves. Aquatic Ecosystem Health and Management Volume 03, Issue 4, p. 561-570, 2000. 
SCHAEFFER-NOVELLI, Y.; CONTRÓN-MOLERO, G. SOARES, M.L.G. Mangroves as indicators of sea-level change in the muddy coasts of the world. In: Wang, Y. and Healy, T. (eds.), Muddy Coasts. United Kingdom: Elsevier, pp. 245-262, 2002.

SCHAEFFER-NOVELLI, Y.; COELHO JR, C.; TOGNELLA-DE-ROSA, M. Manguezais. Ática, 1ed, São Paulo: 2004

SOARES, M.L.G. A conceptual model for responses of mangrove forest to sea level rise. Journal of Coastal Research, especial issue, p. 267271, 2009.

SOLOMON, S. et al. (Eds.), Climate Change: The Physical Science Basis. Contribution of Working Group I to the Fourth Assessment Report of the Intergovernmental Panel on Climate Change. Cambridge University Press, Cambridge, United Kingdom and New York, NY, USA, 2007.

SOLOMON, $\mathrm{S}$ et al. Irreversible Climate Change Due to Carbon Dioxide Emissions. Proct. Natl. Acad. Sci. U.S.A. 106 (6): p. 1704-1709, 2010.

SOUZA-FILHO, P.W.M.S. Costa de macromaré da Amazônia: Cenários morfológicos, mapeamento e quantificação de áreas usando dados de sensores remotos. Revista Brasileira de Geofísica 23 (4): p. 427-435, 2005.

SPALDING, M.; KAINUMAAND, M.; COLLINS, L. World mangrove atlas. London, Washington D. C. ISBN 978-1-84407-657-4. 319 pp, 2010. 